Recepción: 10 / 03 / 2018

Aceptación: 19 / 04 / 2018

Publicación: 05 / 06 / 2018

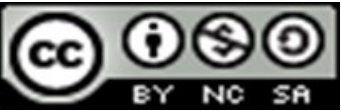

Ciencias de la Computación
Business Informático

\section{Business Computer}

Computador de negócios
Artículo de Investigación
Jasmin A. Saltos-Gómez ${ }^{\text {I }}$ ing.jasycar@gmail.com

Correspondencia: ing.jasycar@gmail.com

I Ingeniera en Sistemas y Tecnologías de la Información, Docente de la Universidad Luis Vargas Torres de Esmeraldas, Esmeraldas, Ecuador. 


\section{Resumen}

Cada día que pasa es más fácil darse cuenta que en esta era del mundo digital, que estamos experimentando más aceleradamente desde hace un par de décadas, cualquier emprendimiento de negocio vinculado a la informática y la computación, entiéndase software o hardware, tiene muchas posibilidades de convertirse en una actividad muy lucrativa. Cada vez más, la tecnología forma parte de nuestro día a día, tanto a nivel personal como profesional, inclusive, el surgimiento de más y nuevas profesiones relacionadas con esta temática, frecuentemente son más comunes, puesto que por la vorágine informativa que se produce tras el constante uso y desarrollo de equipos y soportes informáticos, estos profesionales se vuelven imprescindibles en el mismísimo proceso evolutivo continuo en nuestra sociedad. Esta es una investigación de naturaleza documental a nivel descriptivo, a través de la cual se logró analizar un determinado cúmulo de información al que se accedió mediante el uso de los motores de búsqueda conocidos como Google Académico ${ }^{\circledR}$ y Google ${ }^{\circledR}$, herramientas que condujo a la consulta de revistas científicas, trabajos de investigación de tercer y cuarto nivel, portales web, artículos especializados, entre otros materiales de interés, con los que se ha podido desarrollar el tema del Business Informático (Negocio Informático). Dentro de ese contexto, el presente trabajo se ha enfocado en definir y describir cuáles han sido sus orígenes, para luego exponer en líneas generales la amplia visión e implicación que hoy día se tiene sobre este tipo de negocios, todo ello con la finalidad de aportar un material actualizado haciendo énfasis en la tendencia actual en esta materia, hallada tras la mencionada investigación realizada, para finalmente concluir haciendo referencia a las novedades que en la actualidad se presentan como vertientes de dicho negocio.

Palabras clave: tecnología; equipos; soportes; software; hardware.

\section{Abstract}

Every day that passes is easier to realize that in this era of the digital world, which we are experiencing more rapidly for a couple of decades, any business venture linked to computing and computing, software or hardware, has many possibilities of become a very lucrative activity. Increasingly, technology is part of our day to day, both personally and professionally, including the emergence of more and new professions related to this issue, are often more common, 
because of the whirlwind of information that occurs after the constant use and development of computer equipment and supports, these professionals become essential in the very continuous evolutionary process in our society. This is a documentary research on a descriptive level, through which it was possible to analyze a certain amount of information that was accessed

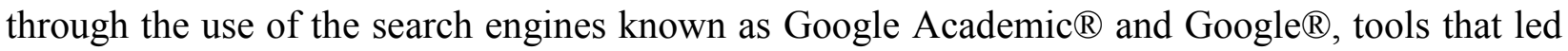
to the consultation of scientific journals, research work of third and fourth level, web portals, specialized articles, among other materials of interest, with which it has been possible to develop the topic of Computer Business (Computer Business). Within this context, the present work has focused on defining and describing what its origins have been, to then outline in general terms the broad vision and implication that this type of business has today, all with the purpose of contributing an up-to-date material emphasizing the current trend in this area, found after the aforementioned research, finally concluding with reference to the novelties that are currently presented as aspects of said business.

Keywords: technology; equipment; supports; software; hardware.

\section{Resumo}

Cada dia que passa é mais fácil de perceber que, nesta era de mute digitais, estamos experimentando mais rapidamente por um par de décadas atrásqualquer empreendimento ligado à tecnologia da informação e computação, entender software ou hardware, tem muitas possibilidades tornar-se uma atividade muito lucrativa. Cada vez mais, a tecnologia faz parte do nosso dia a dia, tanto pessoal quanto profissionalmente, incluindo o surgimento de mais e novas profissões relacionadas a essa questão, muitas vezes são mais comuns, por causa do turbilhão de informações que ocorre após o constante uso e desenvolvimento de equipamentos e suportes de informática, estes profissionais tornam-se essenciais no processo evolutivo muito contínuo em nossa sociedade. Trata-se de uma pesquisa documental em nível descritivo, através da qual foi possível analisar uma determinada quantidade de informações acessadas por meio dos mecanismos de busca conhecidos como Google Acadêmica ${ }^{\circledR}$ e Google ${ }^{\circledR}$, ferramentas que levaram ao consulta de revistas científicas, trabalhos de pesquisa de terceiro e quarto nível, portais web, artigos especializados, entre outros materiais de interesse, com os quais tem sido possível desenvolver o tema do Negócio de Computadores (Computer Business). Dentro deste 
contexto, este trabalho tem se concentrado em definir e descrever o que eram as suas origens, em seguida, expor amplamente a visão ampla e envolvimento que hoje você tem esse tipo de negócio, tudo com o objectivos de proporcionar um material atualizado com ênfase em tendências atuais nesta área, encontrados após essa investigação conduzida, para finalmente concluir, referindo-se às inovações que hoje são apresentados como aspectos do negócio.

Palavras chave: tecnologia; equipamento; suporta; software; hardware.

\section{Introducción}

De acuerdo a la idea de Molina \& Gallardo (2012), en los últimos 50 años se han materializado importantes avances referentes a las Tecnologías de la información y la Comunicación, lo que se puede destacar con especial interés respecto a dichas tecnologías es que se da una marcada tendencia a su miniaturización y multiplicidad de capacidades, lo que ha permitido a sus usuarios la capacidad de desarrollar múltiples habilidades, y además de esto e igualmente importante, está el hecho de la constante tendencia a la baja de los precios, favoreciendo así la accesibilidad para todos. Pudiera afirmarse que la evolución más significativa en dicha materia tecnológica ha sido protagonizada por los dispositivos móviles que logran ofrecer un vasto de soluciones, concretamente en cualquier campo del conocimiento y que otorga el poder y asistencia para la mejor toma de decisiones de los directivos de una empresa, por ejemplo. Como bien pudiera deducirse, a la par de dicha evolución se presenta el fenómeno de la globalización, en la que claramente puede observarse la tendencia de la llamada economía del conocimiento, en la que el principal activo para una organización empresarial es lo intangible, es decir, el conocimiento para obtener resultados tangibles o materiales previamente establecidos. (pp. 440-441)

En el marco de este contexto es que se pretende desarrollar la presente investigación pues, para nadie es un secreto que tras la loable creación del ordenador personal y los subsiguientes sistemas operativos, siempre ha existido un importante negocio, que no sólo ha convertido a sus fundadores en multimillonarios y hasta filántropos, sino que ha abierto las puertas a un mundo de posibilidades de generar tanto ideas como ingresos económicos, bien sea por la creación de productos como por la prestación de servicios asociados.

En concordancia con la postura de representantes de Emprende Pyme (2016), se logra asimilar el hecho de que cada día que pasa es más fácil darse cuenta que en esta era del mundo digital, que 
estamos experimentando, más aceleradamente desde hace aproximadamente un par de décadas, cualquier emprendimiento de negocio vinculado a la informática y la computación, entiéndase software o hardware, tiene muchas posibilidades de convertirse en una actividad muy lucrativa. Cada vez más, la tecnología forma parte de nuestro día a día, tanto a nivel personal como profesional, inclusive, el surgimiento de más y nuevas profesiones relacionadas con esta temática, frecuentemente son cada vez más comunes, puesto que la vorágine informativa que se produce tras el constante uso y desarrollo de equipos y soportes informáticos, hace que estos profesionales se vuelvan imprescindibles en el mismísimo proceso evolutivo de nuestra sociedad.

El objetivo de esta investigación está centrado en desarrollar el tema bajo una visión general, sin entrar en mayores detalles que serían propios de un discernimiento más profunda, específica y sistemático, ya que la idea es aportar un material que describa lo que en la actualidad se puede describir como negocio informático, básicamente entendido como la oferta de productos y servicios asociados al uso y manejo, tanto de equipos informáticos y computacionales como de sus propios sistemas operativos por parte de usuarios convencionales y especializados.

Al concluir este trabajo, se hará mención al negocio informático que, a criterio del investigador, se consideró reciente y frecuente tras la propia búsqueda e investigación del tema central aquí planteado, quedando a juicio del lector aceptarlo o rechazarlo como definitivo.

\section{Materiales y Métodos}

En el presente estudio se han desarrollado esencialmente en el marco de la Tecnología de la Comunicación e Información, ya que a criterio propio se considera como pilar fundamental del Negocio Informático (Business Informático). El propósito fue centrar los esfuerzos en indagar sobre las novedades al respecto, sin dejar de un lado aspectos tan fundamentales tales como su definición, tipos y actualidad en relación al tema del negocio informático, entre otros aspectos de interés.

En base a tales fines se realizó una investigación de tipo documental a nivel descriptivo, basada en fuentes especializadas, accedidas de manera digital, recabando la información necesaria mediante los motores de búsqueda Google Académico ${ }^{\circledR}$ y Google ${ }^{\circledR}$, garantizando el principio de actualidad y veracidad del contenido seleccionado. Se consultaron diversos documentos electrónicos tales como, revistas virtuales especializadas, artículos científicos, libros electrónicos 
y demás fuentes de información en el área de informática, computación, negocios, gerencia y demás temas conexos, los cuales se encontraban alojados en páginas web, repositorios digitales y otras bases de datos, de carácter formal y profesional.

Respecto a la metodología aplicada, Ríos (2017), afirma que la investigación documental “... recurre a la consulta de documentos para obtener sus resultados”. En cuanto a la profundidad de la misma, explica que la investigación es considerada a nivel descriptivo cuando “...busca encontrar las características, comportamiento y propiedades del objeto de estudio, ya sea en el presente o en el futuro..." (p.81).

\section{Resultados}

Un aspecto que indiscutiblemente ha marcado el Business Informático (Negocio Informático) ha sido la innovación, por ello se considera importante iniciar el desarrollo de este trabajo investigativo ofreciendo una definición de dicho término.

Ahumada \& Perusquia (2016), tomando como referencia los aportes al respecto de (Chesbrough, 2011) y (Chesbrough, 2010), la definen como:

Las acciones u operaciones que promueven la mejora de cualquiera de los productos o servicios que produce la empresa, pudiendo ser estos de tipo tecnológico, operacional, administrativo o de estrategia empresarial. Así mismo son las políticas de promoción que la empresa tenga para llevar a cabo valoración de procesos, de productos, de personas en la organización y de acumulación o concentración de información en sus procesos productivos.

\section{Negocio Informático Global}

Al interpretar el grueso de la información obtenida luego de una consulta fundamentada en el principio de la vigencia y pertinencia, puede llegarse a afirmar que el negocio informático básicamente puede entenderse globalmente desde una perspectiva compuesta de dos elementos fundamentales y correlacionados, como lo son: el software y el hardware, alrededor de los cuales gira este negocio informático. En este contexto cabría destacar, por ejemplo, lo expuesto por Espinoza \& Gallegos (2017), quienes hacen referencia a las Tecnologías de la Información (TI). 
Estos autores, a su vez haciendo referencia a técnicas (Laudon y Laudon, 2012, pág.165), explican que la infraestructura de las TI está compuesta por un conjunto de "dispositivos físicos y aplicaciones de software requeridas para operar toda la empresa", a esto bien pudiera agregarse que hoy día ya no es exclusivo de "las empresas" porque, individualmente, se puede acceder a dichos dispositivos y aplicaciones para uso personal o comercial, mejor dicho, de negocio propio. Continúan exponiendo que dicha infraestructura, adicionalmente, "es conceptualizada como un conjunto de servicios a nivel empresarial presupuestado por la gerencia, que abarca las capacidades tanto humanas como técnicas".

Los mismos, esta vez citando a (Pressman, 2010, pág. 2) dicen que:

El software de computadora es una producción intangible que contiene una secuencia de pasos o instrucciones que cumplen con una funcionalidad para un determinado tipo de equipo de cómputo. El software gana realce ya que sigue siendo la tecnología más importante en la escena mundial. Hace 50 años, nadie hubiera podido predecir que el software se convertiría en una tecnología indispensable para los negocios, ciencias e ingeniería, ni que permitiría la creación de tecnologías nuevas, por ejemplo, ingeniería genética y nanotecnología; la ampliación de tecnologías ya existentes como las telecomunicaciones y el cambio radical de tecnologías antiguas como es la industria de la impresión. El crecimiento exponencial previsto en la producción de datos será el resultado de un aumento en el número de instrumentos que registran mediciones de entornos y procesos físicos. (párr. 2)

Atendiendo a lo antes expuesto, entonces es conducente afirmar que es el marco de ese contexto que se materializa lo que hoy día se entiende por Business Informático (Negocio Informático).

\section{La evolución del negocio de la informática}

Serradilla (2013), ha mencionado que tras algunos cambios en el sector informático se viene dando cada vez con mayor auge un modelo de negocio informático caracterizado por el desarrollo del mercado de aplicaciones móviles, y ejemplifica que «stores» como Apple ${ }^{\circledR}$ y Google ${ }^{\circledR}$ ofrecen la posibilidad de que desarrolladores, de manera sencilla e independiente, puedan publicar sus ideas. Así mismo afirma que, por el auge de la red, ha sido posible el impulso de novedosas ideas, claro está, con un costo algo elevado y con una cierta (probablemente pequeña) probabilidad de éxito. Además, revela que tras el impulso que 
proporcionan determinados sectores de la Universidad y algunas empresas (al menos en España, contexto geográfico en que se ubica el autor), se viene materializando un cambio de enfoque, situación lo que inspira a cada vez más estudiantes a independizarse e intentarlo dentro del negocio informático, por cuenta propia, y bien sea que alcancen o no el éxito, en esa etapa siempre obtendrán una experiencia invaluable para su consecuente desarrollo profesional.

\section{Realidades sobre el Emprendimiento Informático}

Hace relativamente poco (Cárdenas, Peralta, Aguirre, Reyes, \& Cervantes, 2016), a través de su trabajo investigativo aportaron informaciones importantes al respecto, por lo que para este tema se consideró valioso compartir.

De dicho trabajo, entre otras cosas, se logra comprender que los emprendedores de las grandes empresas en el mundo se caracterizan por el grado académico, formación que garantizan el crecimiento, desarrollo y futuro de sus empresas, plantean estudios de oferta y demanda, económicos, geográficos, financieros, adicionando planes de negocios estratégicos que les permita llegar al mercado, posesionarse e ir innovando de acuerdo a las necesidades de la sociedad.

Los emprendimientos hoy en día se han convertido en la oportunidad más loable para el mejoramiento de la calidad de vida de los países a nivel mundial, basados en las teorías de la plataforma para el desarrollo de los pueblos, involucrando principios y valores a líderes que buscan plasmar ideas a través de modelos de negocios con visión y crecimiento.

Para finalizar esta parte, igualmente se considera importante mencionar que Cárdenas et al. (2016), citando a (Fracica, 2009), informa que, los países desarrollados, para fomentar el emprendimiento informático entre sus ciudadanos:

Establecen parámetros que garanticen la actividad económica que se emprenda, determinando políticas económicas que respalden la inversión del emprendedor. Además, se observa que en las grandes potencias muchas de las grandes empresas que actualmente son reconocidas y admiradas por su rápido crecimiento y éxito en el mercado como Microsoft, Oracle y Sun MicroSystem, en Estados Unidos, Skype, Movistar y Tom Tom, en Europa, en sus comienzos recibieron el apoyo del capital de riesgo. Así mismo, el capital de riesgo ha sido fundamental para que Estados 
Unidos y otros países adquieran ventajas competitivas en el desarrollo de nuevas industrias como la biotecnología, software y semiconductores. (p.156)

De manera particular el mismo autor refiere que "Los emprendimientos en tecnología deben ser originalmente dinámicos, innovadores para mantenerse en los mercados a nivel mundial", teniendo presente que en la globalización esto es un factor predominante y medio conductor que propicia la competitividad entre pequeños y grandes empresarios, y esto vienen siendo los actores que "toman decisiones para fortalecer productos a servicios, asignando un valor agregado que permita competir en el mercado" (p. 156)

\section{Tendencias}

Con la intención de ir culminando el desarrollo de este trabajo investigativo, en esta sección se pretende informar sobre los enfoques que se vienen dando en la región en materia de negocio informático, para ello a continuación se describen los aspectos más resaltantes de un artículo especializado de Computerworld Colombia (2018), que ejemplifica especialmente algunas vertientes que proveedores de productos y servicios del negocio informático en América Latina se han estado enfocando.

En esa publicación afirman, entre otras cosas que en las empresas latinoamericanas se está produciendo un cambio en el enfoque del gasto en tecnología, con el objetivo de "optimizar el uso de aceleradores digitales de transformación para mejorar sus actividades comerciales" y tales estrategias "están creando oportunidades significativas en las áreas de TI y nube".

Resulta que, a pesar de las ofertas innovadoras propuestas, por ejemplo, por empresas proveedoras de servicios, no logran calar debido a dos factores que contravienen sus intenciones, como lo son los capitales financieros (por falta de ingresos) y humanos (por falta de mano de obra especializada). Sin embargo, en el mismo extracto informático se deja ver claramente que no todo son reveses, pues como punto positivo y hacen referencia al aporte de Sosa (2018) quien entre otras cosas comentó que:

Los proveedores se han vuelto participantes activos en segmentos del mercado y la industria en los que antes no tenían presencia, y ya comenzaron la transición de las grandes corporaciones a 
las pequeñas y medianas empresas, además de diversificar su portfolio de servicios para incrementar los ingresos. (párr.4)

Ahora bien, tomando como base al mismo artículo especializado, se puede agregar que las siguientes tendencias son las que marcaran la pauta en la expansión de los negocios de TI en lo que resta de 2018, y son:

- Externalización de TI: Los modelos de financiación colectiva (crowdfunding, en inglés) pueden transformar la forma en la que las empresas proveen soporte para redes y escritorio, mediante una reducción de costos que puede resultar atractiva para otras empresas que, de lo contrario, no podrían acceder a este soporte con los modelos previos. Estiman que este mercado sobrepase los USD 13 mil millones a finales de 2018.

- Nube pública; porque continuará incrementándose la cantidad de proveedores de servicios de nube administrada y múltiples nubes, lo que alentará nuevas alianzas en el mercado. Para este sector se estima que a fines de este año se alcancen cerca de los USD 4 mil millones.

- Centros de datos; puesto que las soluciones tradicionales de almacenamiento y recuperación en casos de desastre perderán participación en el mercado ante el modelo Infraestructura como Servicio (IaaS) de nube. Las soluciones de automatización, como los centros de datos definidos por software (Software-defined Data Center, en inglés) modernizarán los centros de datos. Las estimaciones para este tipo de negocio oscilan los USD 3 mil millones, hacia fines del año en curso.

- Servicios de seguridad administrada (MSS); el aprendizaje automático (machine learning, en inglés) y el aprendizaje profundo (deep learning, en inglés) serán herramientas poderosas para el mercado de seguridad. A medida que las máquinas aprendan rápidamente y sean capaces de tomar decisiones, podrán prevenir amenazas en lugar de mitigar las consecuencias tras los ataques. Las proyecciones en cifras están estimadas alrededor de USD 620 millones.

- Big Data; las soluciones de IoT (Internet of Thinks, en español, el internet de las cosas) crearán nuevas demandas de soluciones de análisis de datos capaces de integrarse con las 
tecnologías de Inteligencia Artificial (AI) y aprendizaje automático. Hacia finales de 2018 se espera que los números en este ámbito de negocios deberían rondar los USD 785 millones.

En su artículo, López (2018) maravillosamente asegura que:

Los cambios sociales y las posibilidades tecnológicas llevan a continuos avances en los modelos de negocio y las formas de relacionarse entre los usuarios, consumidores y ciudadanos con las organizaciones. La transformación del modelo de relación con el cliente es la prioridad de todas las empresas, para hacer las cosas más fáciles y rápidas.

Finalmente, podría asegurarse que hoy en día el Business Informático (Negocio Informático), está detrás de casi todo lo que nos rodea, puesto que la sociedad actual, por encontrarse en un constante proceso de transformación hacia un mundo cada día más globalizado, (particularmente nuestra región, que incansablemente dirige un lento pero persistente esfuerzo por idear y producir teniendo como ejemplo a las potencias económicas del mundo), seguirá influenciado por los avances tecnológicos que de una manera $\mathrm{u}$ otra siempre van obnubilando tanto al productor de bienes y servicios como al usuario final con las bondades y facilidades que ofrece, es decir, la tendencia siempre será, por el lado de los oferentes del negocio informático, competir por ser el primera o la mejor empresa al ofrecer soluciones tato a otras empresas como a usuarios particulares, que por consiguiente se traduzca en éxito financiero; y por el otro lado, el de los consumidores, siempre estará presente la necesidad de satisfacer esas necesidades a través de productos y servicios que cumplan las exigencias pretendidas.

\section{Conclusiones}

Partiendo del hecho comprobado de que la informática tiene sus bases tras el surgimiento y desarrollo de la computación y sus sistemas, y que la evolución de éstas, como tecnología, ha sido cada vez más poderosa, progresiva y accesible (tanto desde el punto de vista práctico por la facilidad de su uso, como desde punto de vista económico, por la tendencia a la baja en su costo), puede inferirse entonces que el Negocio Informático estará siempre latente y en constante dinamismo con la modernización constante a nivel global, puesto que mientras vayan surgiendo, mejorando y ampliándose todo lo concerniente al software y hardware en nuestra sociedad, ineludiblemente los miembros de ésta nos veremos envueltos y atraídos por las bondades 
intrínsecas que conlleva el uso de la tecnología, lo que a su vez vendría siendo el motor y combustible idóneo para que el negocio subyacente en el ámbito del desarrollo informático se mantenga y evoluciones a lo largo de la existencia de los seres humanos constituidos en sociedad, pues a criterio propio, sin ese elemento fundamental, no habría ni demandantes ni oferentes de esta tecnología informática.

En el mismo orden de ideas, bien cabría destacar a Orozco \& Jacobs (2016) quienes afirmaron que "Las empresas que actualmente se mantienen en constante innovación, deben tomar ventaja de estos recursos y realizar nuevas propuestas dirigidas a su mercado, ya que, aquellas que ignoren estas ventajas se arriesgan a quedar desactualizadas y tal vez, fuera del negocio" (p.174)

Finalmente, y de acuerdo con la opinión de una empresa especializada en el área, se puede asegurar que "Sobrevivir en el sector dependerá de la capacidad de adaptación y de cómo estructurar su plan de negocios". De la misma manera, del mismo portal web experto, fue posible extraer la idea de que no hay acuerdo entre las opiniones de otros especialistas en cuanto a cuál será la evolución de éste mercado, puesto que por una parte se encuentran los que defienden la teoría de los fabricantes y grandes empresas terminaran absorbiendo dicho mercado, pero si eso llegara a ocurrir, se producirá un enorme vacío en relación al servicio y mantenimiento. (100plandenegocios, 2013)

\section{Referencias Bibliográficas}

100plandenegocios. (27 de 06 de 2013). 100plandenegocios.com. Obtenido de 100plandenegocios.com: http://www.100plandenegocios.com/plan-de-negocio-informatica

Ahumada, E., \& Perusquia, J. (2016). Inteligencia de negocios: estrategia para el desarrollo de competitividad en empresas de base tecnológica. Contaduría y Administración, 61(1), 127 - 158. doi: https://doi.org/10.1016/j.cya.2015.09.006

Cárdenas, M., Peralta, M., Aguirre, R., Reyes, M., \& Cervantes, X. (2016). Emprendimientos Informáticos: Una mirada desde la Universidad del Siglo XXI. Didasc@lia: Didáctica y $\begin{array}{llllll}\text { Educación., } & 7(3), & 153 & - & 158 . & \text { Obtenido de }\end{array}$ http://runachayecuador.com/refcale/index.php/didascalia/article/view/1366/780 
Computerworld Colombia. (16 de 07 de 2018). Computerworld Colombia. Obtenido de Computerworld Colombia: https://computerworld.co/nube-y-big-data-son-aceleradores-eninversion-en-america-

latina/?pb:pinned=true\&doing_wp_cron=1531853570.5343708992004394531250

Emprende Pyme. (2016). EmprendePyme.net. Obtenido de EmprendePyme.net: https://www.emprendepyme.net/como-crear-una-empresa-de-informatica.html?pb:pinned=true

Espinoza, M., \& Gallegos, D. (2017). La industria del software en Ecuador: evolución y situación $\begin{array}{lllll}\text { actual. } & \text { Espacios, } & \text { 38(57), } & \text { Obtenido }\end{array}$ http://www.revistaespacios.com/a17v38n57/17385725.html?pb:pinned=true

López, E. (16 de 07 de 2018). interempresas.net. Obtenido de interempresas.net: https://www.interempresas.net/TIC/Articulos/221153-La-firma-en-la-nube-el-puente-entre-elnegocio-y-el-mundo-digital.html

Molina, R., \& Gallardo, J. (2012). Las tecnologías de información y Comunicación: Su evolución y aplicación en los negocios internacionales. INCEPTUM, VII (12), 439 - 466. Obtenido de https://www.inceptum.umich.mx/index.php/inceptum/article/viewFile/258/238?pb:pinned=true

Orozco, I., \& Jacobs, O. (2016). LA NUEVA ERA DE LOS NEGOCIOS: COMPUTACIÓN EN LA NUBE. Télematique, 15(2), 172 - 191. Obtenido de http://ojs.urbe.edu/index.php/telematique/article/view/114/108

Ríos, R. (09 de 2017). www.eumed.net. (E. Grupo de investigación (SEJ 309) eumed.net de la Universidad de Málaga, Ed.) Obtenido de www.eumed.net: http://www.eumed.net/libros/libro.php?id=1662

Serradilla, F. (18 de 10 de 2013). Blogthinkbig.com. Obtenido de Blogthinkbig.com: https://blogthinkbig.com/negocio-de-la-informatica-espana

Sosa, R. (2018). Nube y Big Data son aceleradores en inversión en América Latina. (R. d. Colombia, Entrevistador) Obtenido de https://computerworld.co/nube-y-big-data-sonaceleradores-en-inversion-en-americalatina/?pb:pinned=true\&doing_wp_cron=1531853570.5343708992004394531250 